\title{
Emulation of dynamic simulators with application to hydrology
}

\author{
David Machac ${ }^{\mathrm{a}, \mathrm{b}, *}$, Peter Reichert ${ }^{\mathrm{a}, \mathrm{b}}$, Carlo Albert $^{\mathrm{a}}$ \\ ${ }^{a}$ Eawag, Swiss Federal Institute of Aquatic Science and Technology, Department of Systems Analysis, Integrated \\ Assessment and Modelling, 8600 Dübendorf, Switzerland \\ ${ }^{b}$ ETH Zurich, Department of Environmental Systems Science, 8092 Zurich, Switzerland
}

\begin{abstract}
Many simulation-intensive tasks in the applied sciences, such as sensitivity analysis, parameter inference or real time control, are hampered by slow simulators. Emulators provide the opportunity of speeding up simulations at the cost of introducing some inaccuracy. An emulator is a fast approximation to a simulator that interpolates between design input-output pairs of the simulator. Increasing the number of design data sets is a computationally demanding way of improving the accuracy of emulation. We investigate the complementary approach of increasing emulation accuracy by including knowledge about the mechanisms of the simulator into the formulation of the emulator. To approximately reproduce the output of dynamic simulators, we consider emulators that are based on a system of linear, ordinary or partial stochastic differential equations with a noise term formulated as a Gaussian process of the parameters to be emulated. This stochastic model is then conditioned to the design data so that it mimics the behavior of the nonlinear simulator as a function of the parameters. The drift terms of the linear model are designed to provide a simplified description of the simulator as a function of its key parameters so that the required corrections by the conditioned Gaussian process noise are as small as possible. The goal of this paper is to compare the gain in accuracy of these emulators by enlarging the design data set and by varying the degree of simplification of the linear model. We apply this framework to a simulator for the shallow water equations in a channel and compare emulation accuracy for emulators based on different spatial discretization levels of the channel and for a standard non-mechanistic emulator. Our results indicate that we have a large gain in accuracy already when using the simplest mechanistic description by a single linear reservoir to formulate the drift term of the linear model. Adding some more reservoirs does not lead to a significant improvement in accuracy. However, the transition to a spatially continuous linear model leads again to a similarly large gain in accuracy as the transition from the non-mechanistic emulator to that based on one reservoir.
\end{abstract}

Keywords: emulation, surrogate modelling, hydrological models

\section{Introduction}

In scientific or engineering practice, we often encounter situations where we need to run a computationally complex simulator repeatedly for different inputs (e.g. when doing sensitivity analysis or statistical inference of simulator parameters). This can become infeasible due to excessive computational requirements.

As a workaround to this problem, we can employ a method called emulation, which replaces the simulator by a faster surrogate model, called the emulator, which is an approximation to the simulator [1]. We may construct the emulator as a Gaussian process with empirically specified mean and variance

\footnotetext{
${ }^{*}$ Corresponding author

Email address: david.machac@eawag.ch (David Machac)
} 
functions and condition this process on $n$ sets of design data. Design data are sets of pairs of inputs (or parameter vectors) and corresponding outputs of the simulator, which are required to condition the emulator. After conditioning the stochastic process to the design data, we get an approximation to the simulator outputs for inputs that are not contained in the design data. This basic method of emulation, which does not take into account our knowledge about the simulator, has been developed mainly over the past two decades (e.g. $[2-5]$ ).

Several methods have been proposed to emulate simulators with time series output (dynamic simulators). The two simplest approaches are to treat time as another input [6], or to emulate the output at all time points jointly as a multivariate output of the emulator (time as output) [7]. These naïve techniques may be adequate for short time series. However, they can either become slow or can run into numerical problems when the time series are long or the time steps are densely spaced. Three approaches have been suggested to cope with these problems within the framework of constructing an emulator by conditioning stochastic processes (see the review by Castelletti [8] for conceptually different approaches). Functional approaches to the emulation of dynamic models are to write the output of the simulation model as a series of local or global basis functions over time and emulate the coefficients of this series as functions of the model inputs $[9,10]$. If the model output at one time point depends only on the output at the previous time point, we can emulate the transfer function from one time point to the next $[11,12]$. Finally, the strategy of using a Gaussian process as an innovation term between two successive time points in a dynamic stochastic model was proposed by Liu and West [13].

None of the approaches mentioned above makes use of the knowledge of the simulator mechanisms. This has the advantage that these emulators are very generally applicable. On the other hand, using this knowledge may increase the accuracy of emulation even without extending the design data (which may be computationally demanding). With this motivation, a novel approach, called mechanism-based emulation, was suggested that is based on approximately representing the underlying mechanisms of a simulator by a simplified, linear, stochastic model, the noise term of which is formulated as a Gaussian process of the parameters or inputs to be emulated. This stochastic model is then conditioned to the design data, so that the conditioned noise term corrects dynamically for the errors of the linear model and provides interpolation for new parameters or inputs [14, 15]. As the Gaussian process, after conditioning, corrects the linear model to mimic the behavior of the nonlinear simulator, this approach works with different underlying linear models. We can expect that a more detailed representation of the mechanisms in the simulator, in particular regarding the dependence on parameters or input, leads to a higher precision of the emulator (given the same design data). On the other hand, the simpler the linear model, the faster the emulator. When constructing such a linear approximation, we have to be aware that this approximation has to be a dynamic, linear model in its state variables for given parameters and input (to facilitate conditioning), but that the coefficients of this model can be nonlinear functions of parameters and input. The better these dependencies are formulated, the less corrections by the Gaussian process are needed. The challenge of constructing the emulator is thus to find the ideal compromise between the number of mechanisms to include in the emulator (this relates to the dimension of the state space and to the dependencies of the coefficients on parameters and input), and emulation speed.

We want to achieve two goals with this paper. First, we further extend the approach by Albert [15] to a continuous-space formulation. Second, we quantify the benefit of incorporating knowledge about the mechanisms underlying the simulator in the approximating linear, stochastic model of the emulator for a specific case study. This case study is based on the diffusive approximation of the shallow water equations, a system of two partial differential equations that describe water flow in pipes or open channels. As a simulator, we use a numerical solution of these equations, implemented in Mathematica. This numerical solution exactly copies a single-channel routing component of the Storm Water Management Model (SWMM) by EPA [16] that is used by engineers to predict the outflow from urban drainage basins during and after rain events. Our reason behind using this model 
is that in our future work, we plan to emulate a complex SWMM model, consisting of a network of channels. We will then also use such a complex model to demonstrate the time gain of emulation. In this case study we will compare emulating this simulator with (i) a mechanistic emulator based on an approximating model in the form of a linear, partial, stochastic differential equation (with a continuous state space; as developed in the first part of the paper), (ii) a mechanistic emulator based on sets of linear, ordinary, stochastic differential equations as developed by Albert [15] (different spatial discretizations leading to discrete state spaces of different dimensions) and (iii) a non-mechanistic emulator. The accuracy of emulation is then investigated as a function of the degree of mechanistic description and of the size of the design data set used for conditioning to quantify the benefit of using our knowledge about the mechanisms of the simulator.

\section{Construction of the emulator}

Before introducing an emulator, we need to define what we mean by a simulator, which in the scope of this paper is a deterministic model. Let $\xi(t, x) \in \mathcal{C}\left(\mathbb{R}_{0}^{+} \times X, \mathbb{R}\right), X \subset \mathbb{R}$, denote the timedependent state ${ }^{1}$ of the system. We consider simulators that are given by a partial differential equation of the form

$$
\dot{\xi}(x, t)=f\left(t, x, \partial_{x}, \xi, \psi\right),
$$

where $\boldsymbol{\psi}=\boldsymbol{\psi}(t, x)$ denotes both inputs (observed) and parameters (to be inferred) of the simulator and $t$ and $x$ denote time and spatial variables, respectively. The output of the simulator, $\left\{y_{i a}\right\}$, is given by a finite set of measurements (denoted by the function $g$ ) of the system state at discrete points $t_{i}$ and $x_{a}$ in time and space, respectively, i.e.,

$$
y_{i a}=g\left(\xi\left(t_{i}, x_{a}\right), \boldsymbol{\psi}\right)
$$

In this paper, we are only concerned with the emulation of outputs (2) as a function of model parameters $\boldsymbol{\psi}$ that are independent of both $x$ and $t$. The construction of the mechanism-based emulator starts with the simplification of eqs. (1) and (2). This step usually requires a simplification of the state space. We will construct emulators based on the full state space (Sect. 2.1) as well as for simplified state spaces that are defined through discretizations of $X$ (Sect. 2.2). Once the (simplified) state space is defined, we need to find a crude but computationally fast approximation of the model equations (1) and (2). For our emulator to be efficient we need to be able to solve these simplified equations analytically. Thus, we require them to be linear in the (simplified) state variables. However, the coefficients of these linear equations are allowed to depend non-linearly on the model parameters $\boldsymbol{\psi}$. The design of the simplified model is case-specific and not unique. This is the disadvantage of our emulator compared to standard Gaussian process emulators. However, if one is willing to invest some work into designing such a simplification, for a given model, a lot of efficiency can potentially be gained (see Sect. 3, for a case study). Note that the simplification need not be very accurate, as it only serves as a prior. All the errors caused by neglecting nonlinearities and state variables are represented by a white noise term that is added to the simplified equations. To correct for the simplification errors, the resulting linear stochastic differential equations are conditioned on $n$ pairs of parameters and outputs (design data) that were calculated with the model equations (1) and (2). This conditioning is achieved through coupling $n+1$ replica of the stochastic linear equations of the simplified model via the noise

\footnotetext{
${ }^{1}$ Both $x$ and the image of $\xi$ can be multi-dimensional. To keep notation simple, we restrict ourselves to a onedimensional setting.
} 
term, which, for each time point, is defined as a standard Gaussian process of the parameters. That is, the closer two parameter sets are the stronger the associated replica are coupled. Once this system of stochastic linear equations is conditioned to the design data (the first $n$ components set-up with the parameters of the design data set), we are left with a fast normal predictor of the output for the $(n+1)$ th parameter set. This predictor derives its knowledge about the model response from both the crude prior and the outputs of the design data, especially from those with similar parameter sets.

\subsection{Continuous state space}

In this subsection, we do not simplify the state space, but build the emulator upon a linear stochastic partial differential equation of the form

$$
\dot{\xi}(t, x)=F\left(t, x, \partial_{x}, \boldsymbol{\theta}\right) \xi(t, x)+b(t, x, \boldsymbol{\theta})+C(\boldsymbol{\theta}) \eta(t, x),
$$

the drift term of which is supposed to be a linear approximation to $(1), \eta(t, x)$ is the white noise process and $C$ its amplitude. The mechanistic part is introduced through the linear model consisting of the linear differential operator $F\left(t, x, \partial_{x}, \boldsymbol{\theta}\right)$ and the function $b(t, x, \boldsymbol{\theta})$. To differentiate from the parameters of the simulator, we will denote the parameters of the emulator as $\boldsymbol{\theta}$.

The state of the coupled system is denoted $\boldsymbol{\xi}:=\left(\xi_{1}, \ldots, \xi_{n+1}\right)$. Furthermore, written without an index, the parameter vector $\boldsymbol{\theta}$ shall henceforth refer to the coupled system, i.e.,

$$
\boldsymbol{\theta} \equiv\left(\boldsymbol{\theta}^{1}, \ldots, \boldsymbol{\theta}^{n+1}, \boldsymbol{\theta}^{\prime}\right),
$$

where $\boldsymbol{\theta}^{\alpha}, \alpha \in\{1, \ldots, n+1\}$ refers to parameters of the emulator that can have different values for each replica. These parameters can be a subset of the model parameters $\psi$ or functions thereof. The parameters $\boldsymbol{\theta}^{\prime}$ are not replica-specific. They refer to parameters that describe the noise process (noise amplitudes and correlation lengths) but also other parameters of the simplified model equations that cannot be defined as functions of the model parameters $\boldsymbol{\psi}$ (see the case study in Sect. 3 for an example).

The noise term of the coupled linear system is expressed as

$$
\mathbf{C}(\boldsymbol{\theta})=\sigma \mathbf{R}(\boldsymbol{\theta}),
$$

where

$$
\left(\mathbf{R R}^{T}\right)^{\alpha \beta}(\boldsymbol{\theta})=\exp \left(-\sum_{l}\left(\frac{\theta_{l}^{\alpha}-\theta_{l}^{\beta}}{\gamma_{l}}\right)^{r}\right)
$$

was chosen in a way that the closer to each other the parameter vectors are, the stronger the coupling is. In our implementation, we use $r=2$. Greek letter indices denote the replica, index $l$ denotes the parameter and $\gamma_{\theta_{l}}$ is the correlation length of parameter $\theta_{l}$. There is a wide choice of correlation functions, an overview is given e.g. in [17]. We have chosen the squared exponential correlation function for its smoothness. However, in an application to a real problem, where the dimension of the covariance matrix is large, one can benefit from a correlation function with compact support (e.g. [18]), as it will make the covariance matrix sparse, which has computational benefits (in matrix multiplication and inversion).

The coupled system is then given by $n+1$ coupled linear stochastical partial differential equations

$$
\dot{\boldsymbol{\xi}}(t, x)=\mathbf{F}\left(t, x, \partial_{x}, \boldsymbol{\theta}\right) \boldsymbol{\xi}(t, x)+\mathbf{b}(t, x, \boldsymbol{\theta})+\mathbf{C}(\boldsymbol{\theta}) \boldsymbol{\eta}(t, x),
$$


where the elements of $\mathbf{F}$ and $\mathbf{b}$ are

$$
F_{\beta}^{\alpha}\left(t, x, \partial_{x}, \boldsymbol{\theta}\right)=\delta_{\beta}^{\alpha} F\left(t, x, \partial_{x}, \boldsymbol{\theta}^{\alpha}, \boldsymbol{\theta}^{\prime}\right) \text { and } b^{\alpha}(t, x, \boldsymbol{\theta})=b\left(t, x, \boldsymbol{\theta}^{\alpha}, \boldsymbol{\theta}^{\prime}\right),
$$

and $\delta_{\beta}^{\alpha}$ is the Kronecker delta, defined as $\delta_{\beta}^{\alpha}=1$ if $i=j$ and $\delta_{\beta}^{\alpha}=0$ otherwise.

The probability density function of the state $\boldsymbol{\xi}(t, x)$ for, $x \in X=\mathbb{R}$, on the space of paths ([19]) $\mathcal{C}\left(\mathbb{R}_{0}^{+} \times \mathbb{R}, \mathbb{R}^{n+1}\right)$ is then (without denoting the dependencies)

$$
\begin{aligned}
P[\boldsymbol{\xi}] & \propto \exp \left(-\frac{1}{2} \int_{0}^{+\infty} \int_{-\infty}^{+\infty}(\dot{\boldsymbol{\xi}}-\mathbf{F} \boldsymbol{\xi}-\mathbf{b})^{\dagger}\left(\mathbf{C C}^{T}\right)^{-1}(\dot{\boldsymbol{\xi}}-\mathbf{F} \boldsymbol{\xi}-\mathbf{b}) \mathrm{d} x \mathrm{~d} t\right) \\
& =\exp \left(-\frac{1}{2} \int_{0}^{+\infty} \int_{-\infty}^{+\infty}\left(\boldsymbol{\xi}-\mathbf{D}^{-1} \mathbf{b}\right)^{\dagger} \mathbf{D}^{\dagger}\left(\mathbf{C C}^{T}\right)^{-1} \mathbf{D}\left(\boldsymbol{\xi}-\mathbf{D}^{-1} \mathbf{b}\right) \mathrm{d} x \mathrm{~d} t\right)
\end{aligned}
$$

where

$$
\mathbf{D}\left(t, x, \partial_{t}, \partial_{x}, \boldsymbol{\theta}\right)=\frac{\partial}{\partial t}-\mathbf{F}\left(t, x, \partial_{x}, \boldsymbol{\theta}\right)
$$

Now, we introduce the operator, which maps from the state space into the finite-dimensional space of observations, $\mathbf{H}: \mathcal{C}\left(\mathbb{R}_{0}^{+} \times \mathbb{R}, \mathbb{R}^{n+1}\right) \longrightarrow \mathcal{O}$ and is defined as

$$
(\mathbf{H}[\boldsymbol{\xi}])_{i a}^{\alpha}=K\left(\boldsymbol{\theta}^{\alpha}, \boldsymbol{\theta}^{\prime}\right) \xi^{\alpha}\left(t_{i}, x_{a}\right),
$$

where $t_{i}$ and $x_{a}$ denote the discrete space- and time-points as introduced above and $K\left(\boldsymbol{\theta}^{\alpha}, \boldsymbol{\theta}^{\prime}\right)$ is an input-dependent scalar that defines a slope which should approximate the function $g$ in (2).

The modelled simulator output is then given by (11) i.e.,

$$
y_{i a}^{\alpha}=(\mathbf{H}[\boldsymbol{\xi}])_{i a}^{\alpha} .
$$

The prior probability distribution of this output is calculated by integrating the joint distribution of outputs and states over all states, and is given by the path integral

$$
\begin{aligned}
P[\boldsymbol{y}] & =\int P[\boldsymbol{\xi}] \delta(\mathbf{H}[\boldsymbol{\xi}]-\mathbf{y}) \mathcal{D} \boldsymbol{\xi} \\
& \propto \int \exp \left(\int_{0}^{+\infty} \int_{-\infty}^{+\infty} \tilde{\boldsymbol{\xi}}^{\dagger}(t, x) \mathbf{D}^{\dagger}\left(\mathbf{C C}^{T}\right)^{-1} \mathbf{D} \tilde{\boldsymbol{\xi}}(t, x) \mathrm{d} x \mathrm{~d} t\right) \delta\left(\mathbf{H}[\tilde{\boldsymbol{\xi}}]-\left(\mathbf{y}-\mathbf{H}\left[\mathbf{D}^{-1} \mathbf{b}\right]\right)\right) \mathcal{D} \tilde{\boldsymbol{\xi}} \\
& \propto \exp \left(-\frac{1}{2}(\mathbf{y}-\mathbf{z})^{T} \Sigma^{-1}(\mathbf{y}-\mathbf{z})\right),
\end{aligned}
$$

where we used the substitution

$$
\tilde{\xi}=\boldsymbol{\xi}-\mathbf{D}^{-1} \mathbf{b}
$$

and introduced the mean and covariance matrix

$$
\mathbf{z}=\mathbf{H}\left[\mathbf{D}^{-1} \mathbf{b}\right] \quad \text { and } \quad \Sigma=\mathbf{H}\left[\mathbf{D}^{-1} \mathbf{C} \mathbf{C}^{T}\left(\mathbf{D}^{\dagger}\right)^{-1}\right] \mathbf{H}^{\dagger},
$$

respectively. In (13), $\mathcal{D} \tilde{\boldsymbol{\xi}}$ denotes the path integral measure ${ }^{2}$ given by the infinite product

\footnotetext{
${ }^{2} \mathrm{~A}$ good resource on this topic is [19].
} 


$$
\mathcal{D} \tilde{\boldsymbol{\xi}}=\prod_{t} \mathrm{~d} \tilde{\boldsymbol{\xi}}(t)
$$

If we now denote

$$
\tilde{\mathbf{z}}=\mathbf{D}^{-1} \mathbf{b}
$$

then calculating the mean of the multivariate normal distribution (13) is equivalent to finding a $\tilde{\mathbf{z}}$ that fulfils

$$
\mathbf{D} \tilde{\mathbf{z}}=\mathbf{b}
$$

Solving (18) for $\tilde{\mathbf{z}}$ yields

$$
\tilde{\mathbf{z}}\left(t^{\prime}, x^{\prime}\right)=\int \mathbf{G}\left(t^{\prime}, x^{\prime}, t, x\right) \mathbf{b}(t, x, \theta) \mathrm{d} x \mathrm{~d} t .
$$

where $\mathbf{G}\left(t^{\prime}, x^{\prime}, t, x\right)$ denotes the Green's function of $\mathbf{D}(t, x)$. The latter is defined as the solution of

$$
\mathbf{D}(t, x) \mathbf{G}\left(t, x, t^{\prime}, x^{\prime}\right)=\delta\left(t-t^{\prime}\right) \delta\left(x-x^{\prime}\right),
$$

where $\delta$ is the Dirac delta function.

We use a similar procedure to calculate the covariance matrix, where, additionally, we need to find the adjoint Green's function $\mathbf{G}^{\dagger}\left(t, x, t^{\prime}, x^{\prime}\right)$ of the operator D. Element-wise, after applying the operator $\mathbf{H}$, the resulting formulae are

$$
\begin{gathered}
z^{\alpha}\left(t_{i}, x_{a}\right)=K\left(\boldsymbol{\theta}^{\alpha}, \boldsymbol{\theta}^{\prime}\right) \int_{0}^{+\infty} \int_{-\infty}^{+\infty} G^{\alpha}\left(t_{i}, x_{a}, t, x\right) b^{\alpha}(t, x) \mathrm{d} x \mathrm{~d} t \\
\Sigma^{\alpha \beta}\left(t_{i}, t_{j}, x_{a}, x_{b}\right)=K\left(\boldsymbol{\theta}^{\alpha}, \boldsymbol{\theta}^{\prime}\right) K\left(\boldsymbol{\theta}^{\beta}, \boldsymbol{\theta}^{\prime}\right)\left(\mathbf{C C}^{T}\right)^{\alpha \beta} \int_{0}^{+\infty} \int_{-\infty}^{+\infty} G^{\alpha}\left(t_{i}, x_{a}, t, x\right) G^{\beta \dagger}\left(t, x, t_{j}, x_{b}\right) \mathrm{d} x \mathrm{~d} t .
\end{gathered}
$$

With the last two formulae and the properties of conditional normal distributions, the mean and covariance matrix of the $(n+1)$ th replica conditioned on the first $n$ replica that correspond to the design data are then given as

$$
\begin{aligned}
& \overline{\mathbf{y}}=\mathbf{z}^{n+1}+\Sigma^{n+1, \alpha}\left(\Sigma^{\prime}\right)_{\alpha \beta}^{-1}\left(\mathbf{y}^{\beta}-\mathbf{z}^{\beta}\right), \\
& \bar{\Sigma}=\Sigma^{n+1, n+1}-\Sigma^{n+1, \alpha}\left(\Sigma^{\prime}\right)_{\alpha \beta}^{-1} \Sigma^{\beta, n+1},
\end{aligned}
$$

where we sum only over $\alpha$ and $\beta$ ranging from 1 to $n$ and the covariance matrix was split according to

$$
\Sigma=\left(\begin{array}{c|c}
\Sigma^{\prime} & \Sigma^{\bullet}, n+1 \\
\hline \Sigma^{n+1, \bullet} & \Sigma^{n+1, n+1}
\end{array}\right)
$$

The dimension of $\bar{\Sigma}$ is $N_{t} N_{x} \times N_{t} N_{x}$, where $N_{t}$ and $N_{x}$ are the numbers of time and space points, respectively, at which we evaluate the simulator. 


\subsection{Discrete state space}

In many applications a simplification of the state space might be necessary to design an efficient mechanism-based emulator. We therefore spatially discretize equation (3) and write

$$
\dot{\boldsymbol{\xi}}(t)=\mathbf{F}(t, \boldsymbol{\theta}) \boldsymbol{\xi}(t)+\mathbf{b}(t, \boldsymbol{\theta})+\mathbf{C}(\boldsymbol{\theta}) \boldsymbol{\eta}(t),
$$

where $\mathbf{F}$ is now a matrix. The argument $x$ is no longer needed as the positions in space are now represented by the components of the vector $\boldsymbol{\xi} \in \mathbb{R}^{m}$, where $m$ is the number of discretization points. The character $\boldsymbol{\xi}$ that used to denote the vector with states of various replica now denotes the state at the points of discretization. The matrix $\mathbf{F}$ and the vector $\mathbf{b}$ are defined in such a way that, in the limiting case $m \rightarrow+\infty$, we recover the simplified model equations from Subsect. 2.1.

The system (26) without the last term on the right hand side is a linear system of non-homogeneous ordinary differential equations, which we can easily solve. The solution at time $t_{i+1}$ if we know the state at time $t_{i}$ is given by

$$
\boldsymbol{\xi}\left(t_{i+1}\right)=\underbrace{\mathcal{P} \exp \left(\int_{t_{i}}^{t_{i+1}} \mathbf{F}(\tau, \boldsymbol{\theta}) \mathrm{d} \tau\right)}_{\mathbf{h}_{i}(\boldsymbol{\theta})} \boldsymbol{\xi}\left(t_{i}\right)+\underbrace{\int_{t_{i}}^{t_{i+1}} \mathcal{P} \exp \left(\int_{t^{\prime}}^{t_{i+1}} \mathbf{F}(\tau, \boldsymbol{\theta}) \mathrm{d} \tau\right) \mathbf{b}\left(t^{\prime}, \boldsymbol{\theta}\right) \mathrm{d} t^{\prime}}_{\mathbf{k}_{i}(\boldsymbol{\theta})},
$$

where $\mathcal{P}$ denotes path-ordering. Using the notation from [15], we write (27) as

$$
\boldsymbol{\xi}_{i+1}=\mathbf{h}_{i}(\boldsymbol{\theta}) \boldsymbol{\xi}_{i}+\mathbf{k}_{i}(\boldsymbol{\theta})
$$

The observation operator $\mathbf{H}$ is now a matrix that maps from the $m$-dimensional state space to the $N_{x}$-dimensional output space and is allowed to depend non-linearly on the model parameters $\boldsymbol{\psi}$. Typically, $m$ will be larger than $N_{x}$.

The prior probability distribution is analogous to (13), except that $\mathbf{F}$ in (10) is now a matrix instead of a linear differential operator. The mean of the coupled system of $n+1$ replica is calculated from (28) by recursion

$$
\begin{aligned}
\mathbf{z}_{i}^{\alpha} & =\left(\mathbf{H}^{\alpha}\right) \tilde{\mathbf{z}}_{i}^{\alpha}, \\
\tilde{\mathbf{z}}_{i+1}^{\alpha} & =\mathbf{h}_{i}^{\alpha} \tilde{\mathbf{z}}_{i}^{\alpha}+\mathbf{k}_{i}^{\alpha}, \\
\tilde{\mathbf{z}}_{0}^{\alpha} & =0 .
\end{aligned}
$$

For the covariance matrix of the coupled system we may derive a recursion relation as well. For $j \leq i$, it holds that

$$
\begin{aligned}
\Sigma_{i j}^{\alpha \beta} & =\mathbf{H}^{\alpha} \widetilde{\Sigma}_{i j}^{\alpha \beta}\left(\mathbf{H}^{\beta}\right)^{T}, \\
\widetilde{\Sigma}_{i+1, j}^{\alpha \beta} & =\mathbf{h}_{i}^{\alpha} \widetilde{\Sigma}_{i, j}^{\alpha \beta}, \widetilde{\Sigma}_{00}^{\alpha \beta}=0, \\
\widetilde{\Sigma}_{i i}^{\alpha \beta} & =\widetilde{\Sigma}_{i, i-1}^{\alpha \beta}\left(\mathbf{h}_{i-1}^{\beta}\right)^{\dagger}+\mathbf{g}_{i-1}^{\alpha \beta},
\end{aligned}
$$

where

$$
\mathbf{g}_{i}^{\alpha \beta}=\sigma^{2}\left(\mathbf{R R}^{T}\right)^{\alpha \beta} \int_{t_{i}}^{t_{i+1}} \mathcal{P} \exp \left(\int_{t^{\prime}}^{t_{i+1}} \mathbf{F}\left(\tau, \boldsymbol{\theta}^{\alpha}, \boldsymbol{\theta}^{\prime}\right) \mathrm{d} \tau\right) \mathcal{P} \exp \left(-\int_{t_{i+1}}^{t^{\prime}} \mathbf{F}^{T}\left(\tau, \boldsymbol{\theta}^{\beta}, \boldsymbol{\theta}^{\prime}\right) \mathrm{d} \tau\right) \mathrm{d} t^{\prime} .
$$


To calculate the mean of the emulator, $\overline{\mathbf{y}}$, a recursive formula was developed in [15]. First we have to calculate the covectors

$$
\mathbf{z}_{i \alpha}^{\prime}=\mathbf{T}_{i j}^{\alpha}\left(\mathbf{H}^{\alpha}\right)^{T}\left(\left(\Sigma^{\prime}\right)^{-1}\right)_{\alpha \beta}^{j k}\left(\mathbf{y}_{k}^{\beta}-\mathbf{z}_{k}^{\beta}\right)
$$

where

$$
\mathbf{T}_{i j}^{\alpha}=\left\{\begin{array}{cc}
\mathbf{h}_{i+1}^{\dagger \alpha} \ldots \mathbf{h}_{j-1}^{\dagger \alpha}, & j \geq i+2, \\
\mathbf{1}, & j=i+1, \\
0, & \text { else }
\end{array}\right.
$$

and $\Sigma^{\prime}$ is defined as in (25). The emulation step then consists of calculating

$$
\tilde{\mathbf{y}}_{i+1}=\mathbf{h}_{i}^{n+1} \tilde{\mathbf{y}}_{i}+\mathbf{k}_{i}^{n+1}+\mathbf{g}_{i}^{(n+1) \alpha} \mathbf{z}_{i \alpha}^{\prime}
$$

and applying the observation operator

$$
\overline{\mathbf{y}}_{i}=\left(\mathbf{H}^{n+1}\right) \tilde{\mathbf{y}}_{i} .
$$

The complexity of an emulation step is then of the order $\mathcal{O}\left(N_{t} N_{x} n\right)$ in terms of matrix multiplications of dimension $m \times m$.

\subsection{Parameter estimation}

The parameters of the emulator that are not replica-specific, $\boldsymbol{\theta}^{\prime}$, as introduced in (4), such as correlation lengths $\gamma_{l}$, have to be estimated numerically or analytically. The parameters $\boldsymbol{\theta}^{\prime}$ are, through mean $\mathbf{z}$ and covariance matrix $\Sigma^{\prime}$, parameters of the multivariate normal distribution (13) or its discrete state space equivalent. Parameter estimation is done only on the design data and the vector $\mathbf{z}$ and the matrix $\Sigma^{\prime}$ have then dimensions $n N_{x} N_{t}$ and $n N_{x} N_{t} \times n N_{x} N_{t}$, respectively, for this section. We will set all the correlation lengths $\gamma_{l}$ proportional to the corresponding parameter ranges [14],

$$
\gamma_{l}=\mu \max _{\alpha, \beta}\left|\theta_{l}^{\alpha}-\theta_{l}^{\beta}\right|, \quad \forall l
$$

and estimate their common scaling factor $\mu \in[0,1]$ instead. We determine the $\boldsymbol{\theta}^{\prime}$ parameters by maximizing the likelihood function for the design data, which reads

$$
\mathcal{L}\left(\mathbf{y} \mid \boldsymbol{\theta}^{\prime *}, \sigma^{2}\right)=\frac{1}{(2 \pi)^{n N_{t} N_{x} / 2}\left|\Sigma^{\prime}\right|^{1 / 2}} \exp \left(-\frac{1}{2}(\mathbf{y}-\mathbf{z})^{\dagger}\left(\Sigma^{\prime}\right)^{-1}(\mathbf{y}-\mathbf{z})\right)
$$

where

$$
\boldsymbol{\theta}^{\prime *}=\boldsymbol{\theta}^{\prime} \backslash \sigma^{2}
$$

We separate the $\sigma^{2}$ from the $\boldsymbol{\theta}^{\prime}$ in the argument of $\mathcal{L}$, which allows us to write 


$$
\Sigma^{\prime}=\sigma^{2} \Sigma^{*}
$$

where $\Sigma^{*}$ does not depend on $\sigma^{2}$. Using the last relation, we find the extrema for $\sigma^{2}$ by setting

$$
\frac{\partial \ln \mathcal{L}}{\partial \sigma^{2}}=0
$$

This yields

$$
\sigma^{2}=\frac{1}{n N_{t} N_{x}}(\mathbf{y}-\mathbf{z})^{\dagger} \Sigma^{*-1}(\mathbf{y}-\mathbf{z})
$$

which then reduces the number of unknown parameters by one. Parameter $\sigma^{2}$ is cancelled during the emulation itself, if we are interested in the mean only (notice, how $\sigma^{2}$ enters the recursive relation (38)). However, we still need to calculate it for the estimation of other parameters and for the variance.

The other parameters are estimated by maximizing $\mathcal{L}$. That is equivalent to maximizing

$$
\ln \mathcal{L}\left(\mathbf{y} \mid \boldsymbol{\theta}^{\prime *}\right)=-\frac{1}{2} \ln \left|\Sigma^{\prime}\right|-\frac{1}{2}(\mathbf{y}-\mathbf{z})^{\dagger} \Sigma^{\prime-1}(\mathbf{y}-\mathbf{z})+\text { const. }
$$

The optimization then consists of plugging (45) into (46) and maximizing using an optimization algorithm (e.g. simulated annealing, the FORTRAN implementation [20] of which is used to generate the results shown in this paper). We expect optimal correlation lengths $\gamma$ getting smaller with higher $n$. We therefore have to estimate the parameters for each $n$ separately.

In a typical application, the matrix $\Sigma^{\prime}$ is large, which makes the parameter estimation computationally expensive. For conditioning the emulator, we need just one inversion, but for the estimation, we need to evaluate (46) in each iteration of the optimization algorithm, until it converges. Luckily, to evaluate (46), we do not need to invert $\Sigma^{\prime}$, but we only need to solve the system of linear equations

$$
\Sigma^{\prime} \mathbf{w}=\mathbf{y}-\mathbf{z},
$$

which is computationally less demanding than matrix inversion. The matrix $\Sigma^{\prime}$ is positive definite (from the properties of a non-degenerate covariance matrix), which allows us to use the Cholesky decomposition. Although this algorithm still has cubic complexity, it is easily parallelizable. E.g. in [21], the speed-up of this algorithm resulting from GPU parallelization is one to two orders of magnitude.

If one does not wish to parallelize, new methods have emerged recently, which allow for a matrixfree calculation of the vector $\mathbf{w}$, e.g. the fast multipole method presented in [22] which offers quadratic complexity if the matrix $\Sigma^{\prime}$ is sparse.

\section{Hydrological application}

In this section, we apply the technique from the previous section to specific $F$ and $b$, which will represent a diffusive approximation of the shallow water equations, or more precisely, a simplification thereof. 


\subsection{Simulation model}

Now, we want to model the water flow through a channel, which is governed by the shallow water equations. In our case, we will use their diffusive approximation. The full model shallow water equations are as follows [23-28]:

$$
\begin{aligned}
\frac{\partial A(t, x)}{\partial t}+\frac{\partial Q(t, x)}{\partial x} & =0 \\
\frac{\partial Q(t, x)}{\partial t}+\frac{\partial}{\partial x}\left(\beta \frac{Q^{2}(t, x)}{A(t, x)}\right) & =-g A(x, t)\left(\frac{\partial h(t, x)}{\partial x}+S_{f}(Q, h, x)\right),
\end{aligned}
$$

where equation (48) describes the conservation of mass and equation (49) the conservation of momentum. Variable $A\left[\mathrm{~m}^{2}\right]$ is the cross-section of the water flow, $Q\left[\mathrm{~m}^{3} \cdot \mathrm{s}^{-1}\right]$ is the discharge, $g\left[\mathrm{~m} \cdot \mathrm{s}^{-2}\right]$ is the gravitational acceleration, $S_{f}[-]$ is the friction slope and $h[\mathrm{~m}]$ is the water level with respect to some reference height. The momentum correction factor $\beta[-]$ is often approximated to be unity. If we split the water level $h$ as

$$
h(t, x)=h_{B}(x)+h_{0}(t, x)
$$

where $h_{B}$ is the height of the channel floor with respect to the reference height and $h_{0}$ is the water depth, and express $S_{f}$ with $h_{0}$ rather than $h$, we get, for the conservation of momentum (49),

$$
\frac{\partial Q(t, x)}{\partial t}+\frac{\partial}{\partial x}\left(\frac{Q^{2}(t, x)}{A(t, x)}\right)=-g A(t, x)\left(S_{0}(x)+\frac{\partial h_{0}(t, x)}{\partial x}+S_{f}\left(Q, h_{0}, x\right)\right)
$$

where $S_{0}[-]$ is the channel floor slope. The diffusive approximation neglects the terms on the left hand side of (51). If we express the water depth as a function of the cross-sectional area (using the known channel geometry)

$$
h_{0}=h_{0}(A(x, t), x)
$$

we can replace

$$
\frac{\partial h_{0}(t, x)}{\partial x}=\frac{\partial h_{0}(A, x)}{\partial A} \frac{\partial A}{\partial x}+\frac{\partial h_{0}(A, x)}{\partial x}
$$

in (51). Finally, expressing $S_{f}$ with $A$ rather than with $h_{0}$, we have from (51) and the diffusive approximation

$$
0=S_{0}(x)+S_{f}(Q, A, x)+\frac{\partial h_{0}(A, x)}{\partial A} \frac{\partial A}{\partial x}+\frac{\partial h_{0}(A, x)}{\partial x} .
$$

If we solve this implicit equation for $Q\left(A, \frac{\partial A}{\partial x}, x\right)$, and insert the result into (48), we get

$$
\frac{\partial A}{\partial t}+\frac{\partial Q}{\partial A} \frac{\partial A}{\partial x}+\frac{\partial Q}{\partial x}+\frac{\partial Q}{\partial \frac{\partial A}{\partial x}} \frac{\partial^{2} A}{\partial x^{2}}=0
$$


Note that the explicit dependence of $Q\left(A, \frac{\partial A}{\partial x}, x\right)$ on $x$ is only through the varying slope, $S_{0}(x)$, friction, $S_{f}(Q, A, x)$, and the channel geometry, $h_{0}(A, x)$. If we assume a prismatic channel with uniform slope and friction (what is typical for sewer pipes), there is no explicit dependence of the implicit solution $Q\left(A, \frac{\partial A}{\partial x}, x\right)$ of equation (54) on $x$ and we can drop $\frac{\partial Q}{\partial x}$ in equation (55). We then get

$$
\frac{\partial A}{\partial t}+\frac{\partial Q}{\partial A} \frac{\partial A}{\partial x}=-\frac{\partial Q}{\partial \frac{\partial A}{\partial x}} \frac{\partial^{2} A}{\partial x^{2}}
$$

which is the foundation of our simulation model. The term on the right hand side represents the peak attenuation (diffusion) of the discharge, whereas the second term on the left hand side represents the wave propagation. We are using the Manning-Strickler form of the friction slope [29]

$$
S_{f}=n_{M}^{2} \frac{1}{R^{4 / 3}} \frac{Q|Q|}{A^{2}}
$$

which yields

$$
Q=\frac{1}{n_{M}} A R(A, x)^{2 / 3} \operatorname{sign}\left(S_{0}-\frac{1}{w(A, x)} \frac{\partial A}{\partial x}\right) \sqrt{\left|S_{0}-\frac{1}{w(A, x)} \frac{\partial A}{\partial x}\right|},
$$

where $n_{M}$ is Manning's roughness coefficient, $R(A, x)$ is the hydraulic radius [m] and $w(A, x)$ is the channel width $[\mathrm{m}]$. We use a channel with a rectangular cross section with a constant $w$, which leads to the hydraulic radius

$$
R(A)=\frac{A}{2 \frac{A}{w}+w} .
$$

Boundary and initial conditions. We need to specify initial and boundary conditions for equation (56). We choose a zero-discharge initial condition that, from (58) leads to a cross-sectional area of zero:

$$
A(0, x)=0, \quad \forall x \geq 0 .
$$

The upstream boundary condition is specified for the discharge

$$
Q(t, 0)=\left\{\begin{array}{ll}
0 & t \notin\left(0, T_{\text {pulse }}\right], \\
I & t \in\left(0, T_{\text {pulse }}\right],
\end{array} \quad \forall t \geq 0,\right.
$$

where we use an open interval to prevent the pulse to start right at $t=0$ and thus ensuring compatibility of the boundary condition with (60). This specifies a pulse input of magnitude $I$ and pulse length $T_{\text {pulse }}$.

We model a semi-infinite channel and thus do not need a downstream boundary condition in order to construct the emulator, as becomes evident later on. However, in order to generate the design data, which we do by solving (56) numerically with Mathematica [30] and its function NDsolve, we need a second boundary condition. We use an "open boundary condition",

$$
\left.\frac{\partial A(t, x)}{\partial x}\right|_{x=k L}=0, \quad k>1
$$

where $L$ is a finite distance where we emulate the output later on. We set the boundary condition for $k=2$, as this ensures that, at $L$, the channel behaves approximately like a semi-infinite one. We have tested that by increasing $k$ further, the result at $L$ remain unchanged (only NDSOLVE gets slower). 


\subsection{Emulator for the diffusive approximation of the shallow water equations}

Linear model. We want the linear model to mimic the nonlinear one as good as possible with respect to the dependency on the model parameters. An obvious choice for a linear approximation of model equations (56) and (58), would be a linearisation of (56) around a particular solution. However, simulating a large range of parameters will take us far away from the regime where such an approximation is valid. Instead, based on (56), we are formulating the linear differential equation

$$
\frac{\partial A}{\partial t}+\lambda \frac{\partial A}{\partial x}=\nu \frac{\partial^{2} A}{\partial x^{2}}
$$

and take inspiration from (58) to formulate reasonable dependencies of $\lambda$ and $\nu$ on the parameters to be emulated. We choose

$$
\lambda=k_{0} \frac{w^{2 / 3} \sqrt{S_{0}}}{n_{M}} \quad \text { and } \quad \nu=k_{1} \frac{w^{5 / 3}}{n_{M} \sqrt{S_{0}}}
$$

with

$$
S_{0}=\frac{L}{d},
$$

where $d[\mathrm{~m}]$ is the height difference between both ends of the channel and $L[\mathrm{~m}]$ is the length of the channel. In equation (64), $k_{0}$ and $k_{1}$ are dimensionless empirical parameters of the emulator. This parameterization uses both, the correct dependence on the Manning-Strickler coefficient $n_{M}$, and the asymptotic dependence on the slope $S_{0}$, as derived from (58). Furthermore, we have multiplied the coefficients with appropriate powers of $w$ to be left with two dimensionless calibration parameters $k_{0}$ and $k_{1}$. The choice we have made in this linear approximation is our "educated guess" and not the result of a systematic optimization. As these relationships are only used for the prior of the emulator that will be conditioned to the design data, other parameterizations would also lead to a valid emulator. However, capturing the main dependences on the emulated parameters in the parameterization (64) is expected to lead to an emulator with a better performance. A poor choice would require a large number of design data to correct for the inadequate parameterization made in this step. To avoid this, one should always test the emulator with a custom simplified model against an emulator with a constant zero prior, which is the default.

Summarizing the parameters we are using, we have

$$
\begin{aligned}
\boldsymbol{\theta}^{\alpha} & =\left(n_{M}^{\alpha}, w^{\alpha}, L^{\alpha}, d^{\alpha}\right)^{T}, \forall \alpha \in\{1, \ldots, n+1\} \quad \text { and } \\
\boldsymbol{\theta}^{\prime} & =\left(k_{0}, k_{1}, \mu, \sigma^{2}\right)^{T} .
\end{aligned}
$$

\subsubsection{Continuous space}

Writing (63) in the form of eq. (3) we get

$$
F^{\alpha}=-\lambda^{\alpha} \frac{\partial}{\partial x}+\nu^{\alpha} \frac{\partial^{2}}{\partial x^{2}}
$$

where

$$
\lambda^{\alpha}=\lambda\left(\boldsymbol{\theta}^{\alpha}, \boldsymbol{\theta}^{\prime}\right) \quad \text { and } \quad \nu^{\alpha}=\nu\left(\boldsymbol{\theta}^{\alpha}, \boldsymbol{\theta}^{\prime}\right) .
$$


The Green's function of the associated linear operator (10) (see, e.g., [31]) reads as

$$
G^{\alpha}\left(t, x, t^{\prime}, x^{\prime}\right)=\frac{1}{2 \pi} \Theta\left(t-t^{\prime}\right) \sqrt{\frac{\pi}{\nu^{\alpha}\left(t-t^{\prime}\right)}} \exp \left(-\frac{\left(x-x^{\prime}-\lambda^{\alpha}\left(t-t^{\prime}\right)\right)^{2}}{4 \nu^{\alpha}\left(t-t^{\prime}\right)}\right),
$$

where $\Theta\left(t^{\prime}-t\right)$ is the Heaviside function. For (24), we further need the adjoint of $G^{\alpha}$, which is given by

$$
G^{\alpha \dagger}\left(t^{\prime}, x^{\prime}, t, x\right)=G^{\alpha}\left(t, x, t^{\prime}, x^{\prime}\right)
$$

Mean function. Having the Green's function, we calculate the mean function from (21). Our observable space now consist of cross-sections at discrete time points and at a fixed $x$. Since the observed variable and the state space variable are identical, the function $K$ is unity, and the mean is given by

$$
z^{\alpha}\left(t_{i}, L^{\alpha}\right)=\int_{0}^{t_{i}} \int_{-\infty}^{+\infty} \frac{1}{2 \pi} \sqrt{\frac{\pi}{\nu^{\alpha}\left(t_{i}-t^{\prime}\right)}} \exp \left(-\frac{\left(L^{\alpha}-x^{\prime}-\lambda^{\alpha}\left(t_{i}-t^{\prime}\right)\right)^{2}}{4 \nu^{\alpha}\left(t_{i}-t^{\prime}\right)}\right) b^{\alpha}\left(t^{\prime}, x^{\prime}\right) \mathrm{d} x^{\prime} \mathrm{d} t^{\prime} .
$$

However, we have the input prescribed through the boundary condition (61) as a flux, not as a crosssection. To account for this, we have to calculate $b^{\alpha}\left(t^{\prime}, x^{\prime}\right)$ from the boundary condition (61). From (63) and (48), we have for the relation between the flow and the cross-section

$$
Q^{\alpha}(t, x)=\lambda^{\alpha} A^{\alpha}(t, x)-\nu^{\alpha} \frac{\partial A^{\alpha}}{\partial x}(t, x)
$$

which, when applied to the boundary condition, yields

$$
Q^{\alpha}(t, 0)=\lambda^{\alpha} A^{\alpha}(t, 0)-\nu^{\alpha} \frac{\partial A^{\alpha}}{\partial x}(t, 0)
$$

where from (72), we have

$$
\begin{aligned}
A^{\alpha}(t, 0) & =\int_{0}^{t} \sqrt{\frac{1}{4 \pi \nu^{\alpha}\left(t-t^{\prime}\right)}} \exp \left(-\frac{\left(\lambda^{\alpha}\right)^{2}\left(t-t^{\prime}\right)}{4 \nu^{\alpha}}\right) b^{\alpha}\left(t^{\prime}\right) \mathrm{d} t^{\prime} \quad \text { and } \\
\frac{\partial A^{\alpha}}{\partial x}(t, 0) & =\frac{\lambda^{\alpha}}{4 \nu^{\alpha}} \int_{0}^{t} \sqrt{\frac{1}{4 \pi \nu^{\alpha}\left(t-t^{\prime}\right)}} \exp \left(-\frac{\left(\lambda^{\alpha}\right)^{2}\left(t-t^{\prime}\right)}{4 \nu^{\alpha}}\right) b^{\alpha}\left(t^{\prime}\right) \mathrm{d} t^{\prime} .
\end{aligned}
$$

Since we have a non-zero input only for $x=0$, the $x^{\prime}$ vanishes from the integrand and we therefore integrate only over $t^{\prime}$. Plugging (75) and (76) as well as (61) into (74) and applying the Laplace transformation we find

$$
b^{\alpha}\left(t^{\prime}\right)=b^{\prime \alpha}\left(t^{\prime}\right)-b^{\prime \alpha}\left(t^{\prime}-T_{p u l s e}\right)
$$

with

$$
b^{\prime \alpha}\left(t^{\prime}\right)=\frac{4 I \exp \left(-\frac{\left(\lambda^{\alpha}\right)^{2} t^{\prime}}{4 \nu^{\alpha}}\right)}{3 \lambda^{\alpha} \sqrt{t^{\prime} \pi}} \operatorname{erf}\left(\frac{\lambda^{\alpha} \sqrt{t^{\prime}}}{2 \sqrt{\nu^{\alpha}}}\right) \Theta\left(t^{\prime}\right) .
$$

The resulting integral over time in the expression for $z^{\alpha}\left(t_{i}, L^{\alpha}\right)$ cannot easily be calculated analytically and we have to resort to numerical integration. 
Covariance matrix. Plugging the Green's function (70) into (22), we get for $t_{j} \leq t_{i}$

$$
\begin{gathered}
\Sigma^{\alpha \beta}\left(t_{i}, t_{j}, L^{\alpha}, L^{\beta}\right)=\left(\mathbf{C C}^{T}\right)^{\alpha \beta} \int_{0}^{t_{\max }} \int_{-\infty}^{+\infty} \Theta\left(t_{i}-t^{\prime}\right) \frac{1}{2} \sqrt{\frac{1}{\pi \nu^{\alpha}\left(t_{i}-t^{\prime}\right)}} \exp \left(-\frac{\left(\left(L^{\alpha}-x^{\prime}\right)-\lambda^{\alpha}\left(t_{i}-t^{\prime}\right)\right)^{2}}{4 \nu^{\alpha}\left(t_{i}-t^{\prime}\right)}\right) \\
\cdot \Theta\left(t_{j}-t^{\prime}\right) \frac{1}{2} \sqrt{\frac{1}{\pi \nu^{\beta}\left(t_{j}-t^{\prime}\right)}} \exp \left(-\frac{\left(\left(L^{\beta}-x^{\prime}\right)-\lambda^{\beta}\left(t_{j}-t^{\prime}\right)\right)^{2}}{4 \nu^{\beta}\left(t_{j}-t^{\prime}\right)}\right) \mathrm{d} t^{\prime} \mathrm{d} x^{\prime} .
\end{gathered}
$$

After integrating the Gaussian integral in $x^{\prime}$, we get

$$
\begin{aligned}
\Sigma^{\alpha \beta}( & \left.t_{i}, t_{j}, L^{\alpha}, L^{\beta}\right) \\
& =\int_{0}^{t_{j}}\left(\mathbf{C C}^{T}\right)^{\alpha \beta} \exp \left(-\frac{\left(L^{\alpha}\right)^{2}}{4\left(t_{i}-t^{\prime}\right) \nu^{\alpha}}-\frac{\left(L^{\beta}\right)^{2}}{4\left(t_{j}-t^{\prime}\right) \nu^{\beta}}+\frac{L^{\alpha} \lambda^{\alpha}}{2 \nu^{\alpha}}+\frac{L^{\beta} \lambda^{\beta}}{2 \nu^{\beta}}-\frac{\left(\lambda^{\alpha}\right)^{2}}{4 \nu^{\alpha}}-\frac{\left(\lambda^{\beta}\right)^{2}}{4 \nu^{\beta}}\right) \\
& \cdot \sqrt{\frac{1}{4 \pi\left(\nu^{\alpha}\left(t_{i}-t^{\prime}\right)+\nu^{\beta}\left(t_{j}-t^{\prime}\right)\right)}} \\
& \cdot \exp \left(-\frac{\left(x_{i}\left(t_{j}-t^{\prime}\right) \nu^{\beta}+x_{j}\left(t_{i}-t^{\prime}\right) \nu^{\alpha}-\nu^{\alpha} \nu^{\beta}\left(t_{i}-t^{\prime}\right)\left(t_{j}-t^{\prime}\right)\left(\lambda^{\alpha}+\lambda^{\beta}\right)\right)^{2}}{4 \nu^{\alpha} \nu^{\beta}\left(t_{i}-t^{\prime}\right)\left(t_{j}-t^{\prime}\right)}\right) \mathrm{d} t^{\prime} .
\end{aligned}
$$

For $t_{j}>t_{i}$, we use the symmetry of the covariance matrix. The resulting integral with respect to $t^{\prime}$ cannot be easily calculated analytically and we therefore have to resort to numerical integration in the implementation.

Recursive formula for the covariance matrix. To reduce the computational burden of calculating the covariance matrix, we derive a recursive formula for this task. One sees that the integrand in (82) can be written as $f\left(t_{i}-t^{\prime}, t_{j}-t^{\prime}\right)$. With a discrete time step $\Delta t$ and a simple substitution $t^{\prime}=\tau+\Delta t$, we write

$$
\begin{aligned}
\Sigma^{\alpha \beta}\left(t_{i+1}, t_{j+1}, L^{\alpha}, L^{\beta}\right) & =\int_{0}^{t_{j+1}} f\left(t_{i+1}-t^{\prime}, t_{j+1}-t^{\prime}\right) \mathrm{d} t^{\prime} \\
& =\int_{0}^{\Delta t} f\left(t_{i}-\tau, t_{j}-\tau\right) \mathrm{d} \tau+\int_{0}^{t_{j}} f\left(t_{i}-\tau, t_{j}-\tau\right) \mathrm{d} \tau \\
& =\int_{0}^{\Delta t} f\left(t_{i}-\tau, t_{j}-\tau\right) \mathrm{d} \tau+\Sigma^{\alpha \beta}\left(t_{i}, t_{j}, L^{\alpha}, L^{\beta}\right),
\end{aligned}
$$

with

$$
\Sigma^{\alpha \beta}\left(\bullet, 0, L^{\alpha}, L^{\beta}\right)=0 .
$$

At each time step, we therefore have to numerically integrate only from 0 to $\Delta t$. Such formula cannot be derived for the mean function, because of the time-varying input $b^{\alpha}\left(t^{\prime}\right)$ that appears in the integrand of eq. (72). 


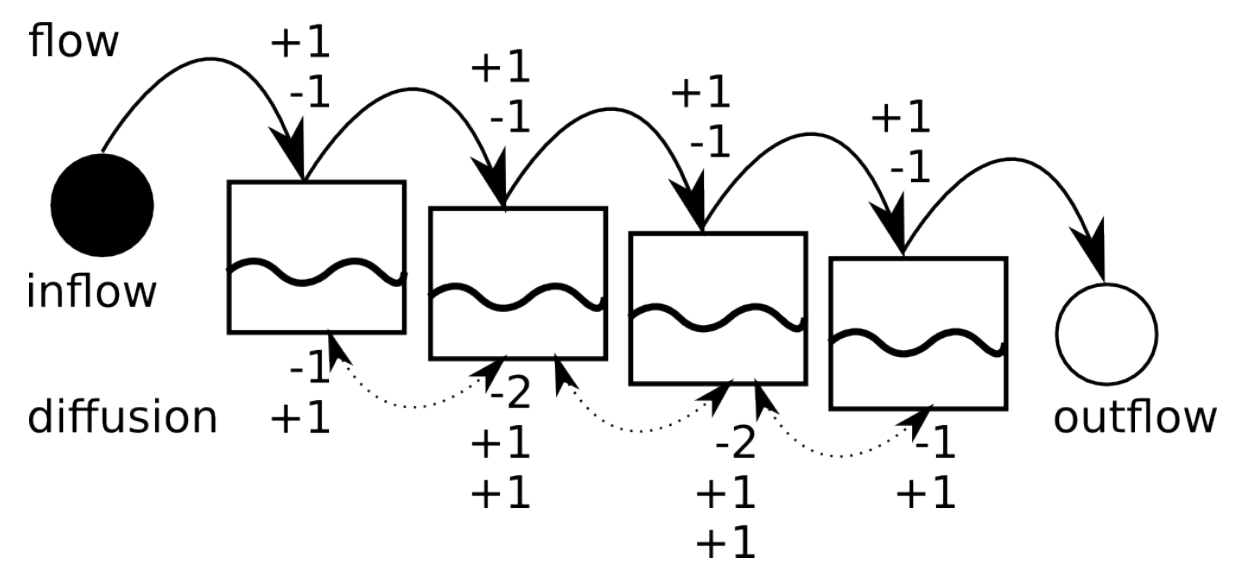

Figure 1: Graphical representation of the discretization of spatial derivatives. Here we have $m=4$. The numbers (if multiplied by $\lambda$ or $\nu$ ) represent the change of mass of water in the respective pipe section. One unit is always added from the previous section or source and one is always lost to the next section or sink due to the flow. Except for the first and last sections, two units are always lost due to diffusion and are distributed to the neighbouring sections.

\subsubsection{Discrete space}

We now discretize the spatial derivatives from (63). Using the spatial discretization for $m$ nodes, we may replace $\lambda \frac{\partial A}{\partial x}$ by

$$
\underbrace{\lambda \frac{m}{L}\left(\begin{array}{ccccc}
1 & 0 & 0 & \cdots & 0 \\
-1 & 1 & 0 & \cdots & 0 \\
0 & -1 & 1 & \cdots & 0 \\
\vdots & \ddots & & & \\
0 & \cdots & 0 & -1 & 1
\end{array}\right)}_{\mathbf{F}_{1}} \mathbf{A}(t)
$$

where the vector $\mathbf{A} \in \mathbb{R}_{+}^{n}$ has the values of $A$ at the $m$ nodes as elements. For the diffusive part, $\nu \frac{\partial^{2} A}{\partial x^{2}}$, we use

$$
\underbrace{\nu \frac{m^{2}}{L^{2}}\left(\begin{array}{ccccc}
1 & -1 & 0 & \cdots & 0 \\
-1 & 2 & -1 & \cdots & 0 \\
0 & -1 & 2 & \cdots & 0 \\
\vdots & \ddots & & & \\
0 & \cdots & 1 & -2 & 1 \\
0 & \cdots & 0 & -1 & 1
\end{array}\right)}_{\mathbf{F}_{2}} \mathbf{A}(t) .
$$

The discretized spatial derivatives have a clear interpretation, as we see in Figure 1. This interpretation is useful, if we want to emulate a network of pipes instead of a single pipe. Instead of bi- and tridiagonal matrices (87) and (88), respectively, we will then get more general matrices. We investigate this approach in our future work, which will apply the theory derived in this paper to a real sewer network case study.

Our linear model in the discrete case is then

$$
\mathbf{F}=-\mathbf{F}_{1}+\mathbf{F}_{2}
$$


In our particular case, $\mathbf{F}$ does not depend on $t$ and we therefore have $\forall i \in \mathbb{N}_{0}^{+}$

$$
\begin{aligned}
\mathbf{h}_{i}^{\alpha} & =\mathbf{h}^{\alpha}=\exp \left(\Delta t \mathbf{F}_{\alpha}\right) \\
\mathbf{k}_{i} & =\int_{t_{i}}^{t_{i+1}} \exp \left(\left(t_{i+1}-t^{\prime}\right) \mathbf{F}_{\alpha}\right) \mathbf{b}\left(t^{\prime}, \boldsymbol{\theta}^{\alpha}\right) \mathrm{d} t^{\prime} \text { and } \\
\mathbf{g}_{i}^{\alpha \beta} & =\mathbf{g}^{\alpha \beta}=\sigma^{2}\left(\mathbf{R R}^{T}\right)^{\alpha \beta} \int_{0}^{\Delta t} \exp \left(t^{\prime} \mathbf{F}_{\alpha}\right) \exp \left(t^{\prime} \mathbf{F}_{\beta}^{T}\right) \mathrm{d} t^{\prime},
\end{aligned}
$$

where

$$
\Delta t=t_{i+1}-t_{i}, \forall i \in \mathbb{N}
$$

Similarly to the continuous case, we again have to deal with the input being prescribed as a flux. Converting it to a cross-section, we get the input

$$
\mathbf{b}\left(t, \boldsymbol{\theta}^{\alpha}\right)=\left(\frac{m}{L^{\alpha}} Q(t, 0), 0, \ldots, 0\right)^{T} .
$$

Since we are interested in the cross-section at the end of the observed system, the observation operator is

$$
\mathbf{H}^{\alpha}=\mathbf{H}=(0, \ldots, 0,1) .
$$

We then calculate the mean and variance of the emulated result by plugging $\mathbf{h}, \mathbf{k}$ and $\mathbf{g}$ into (39) and the preceding equations.

\subsection{Emulation results}

To test the emulator, we created two sets of simulator inputs/outputs, comprising 100 runs each. The parameters of the channel, for each of the $2 \times 100$ runs, are chosen from a uniform distribution within the range of $\pm 20 \%$ around the following (realistic) values: $n_{M}=0.06 \mathrm{~s} / \mathrm{m}^{1 / 3}, w=1 \mathrm{~m}, L=800$ $\mathrm{m}$ and $d=20 \mathrm{~m}$. The input is not varied and is given by a pulse of $Q_{0}=10 \mathrm{~m}^{3} \cdot \mathrm{s}^{-1}$ lasting for 50 minutes.

For the next step, we randomly pick from the first set of inputs/outputs $n$ design data sets and from the second one, we pick randomly 20 data sets for the validation of the emulator. We then run the emulator for each of these 20 sets and calculate the root mean squared error between the emulator and simulator outputs for each of these. This step, picking $n$ design data sets and random 20 test sets, is then repeated 10 times and the average root mean squared error is calculated. This result, for various n's and linear model complexities is shown in Figure 4. This figure furthermore contains results for the non-mechanistic emulator. The non-mechanistic emulator used is a combination of a Gaussian emulator as described in [32] and implemented in the $\mathrm{R}$ package EMULATOR [33], with time as input, and of our proposed emulator with replacing the approximate linear model by the constant zero. The former performs better for less design data sets while the latter performs better for more design data sets. The result we plot, for a non-mechanistic emulator, is then the better of these two, for each $n$.

For each model complexity and each number of design data sets, values of parameters $k_{0}, k_{1}$ and $\mu$ were estimated. The parameter $\sigma^{2}$ is calculated according to (45). Outputs of the linear model for various complexities are shown in Figure 2. 


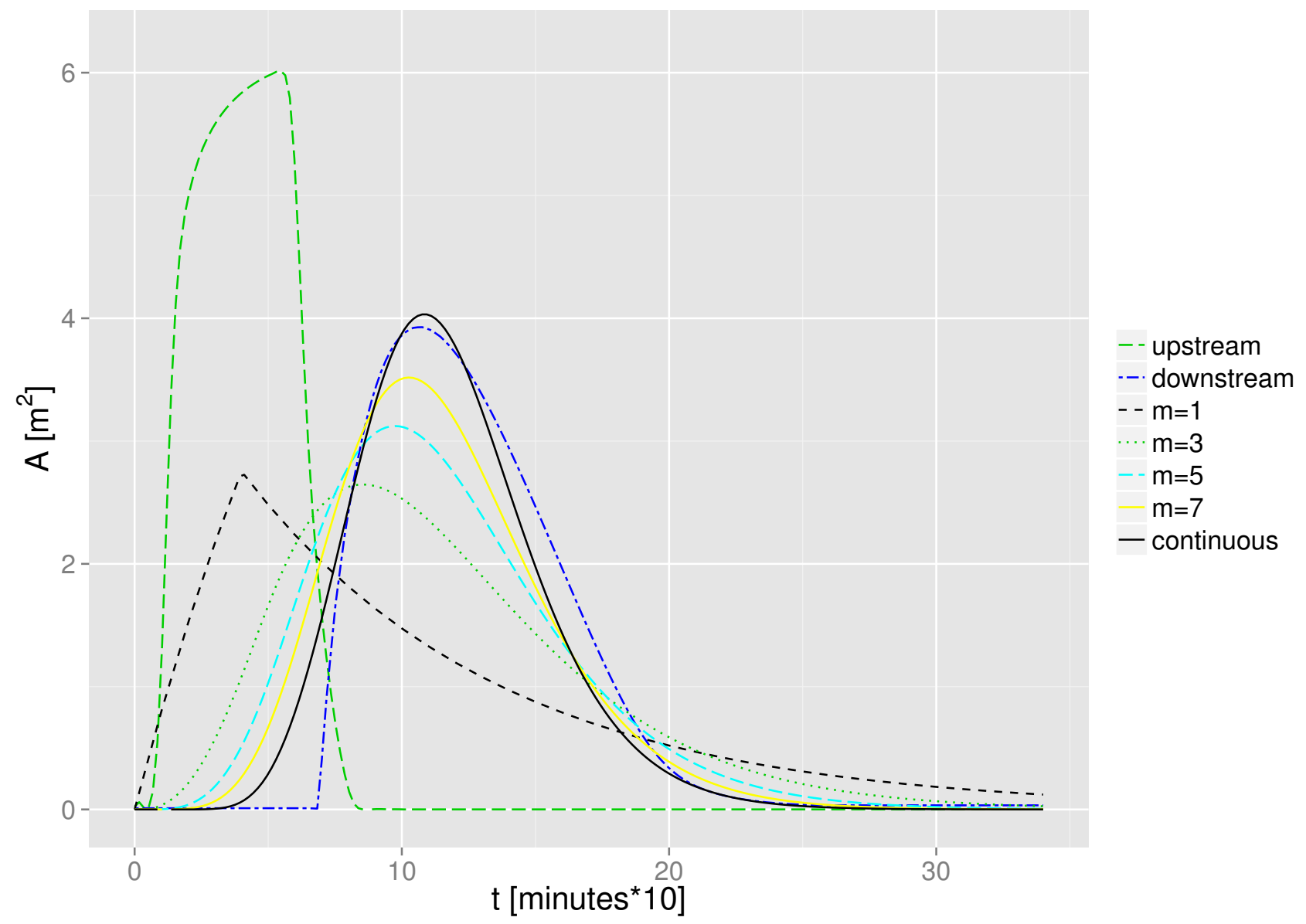

Figure 2: Simulated cross-section $A$ at the inflow and outflow points of the pipe, denoted upstream and downstream, respectively. Remaining curves represent outputs of the linear model, without conditioning to the design data, for various values of $m$, ranging from zero to infinity (the continuous case). We refer to the parameter $m$ as model complexity. To generate these outputs, we have used reference parameter values: $n_{M}=0.06 \mathrm{~s} / \mathrm{m}^{1 / 3}, w=1 \mathrm{~m}, L=800 \mathrm{~m}$ and $d=20$ $\mathrm{m}$. 

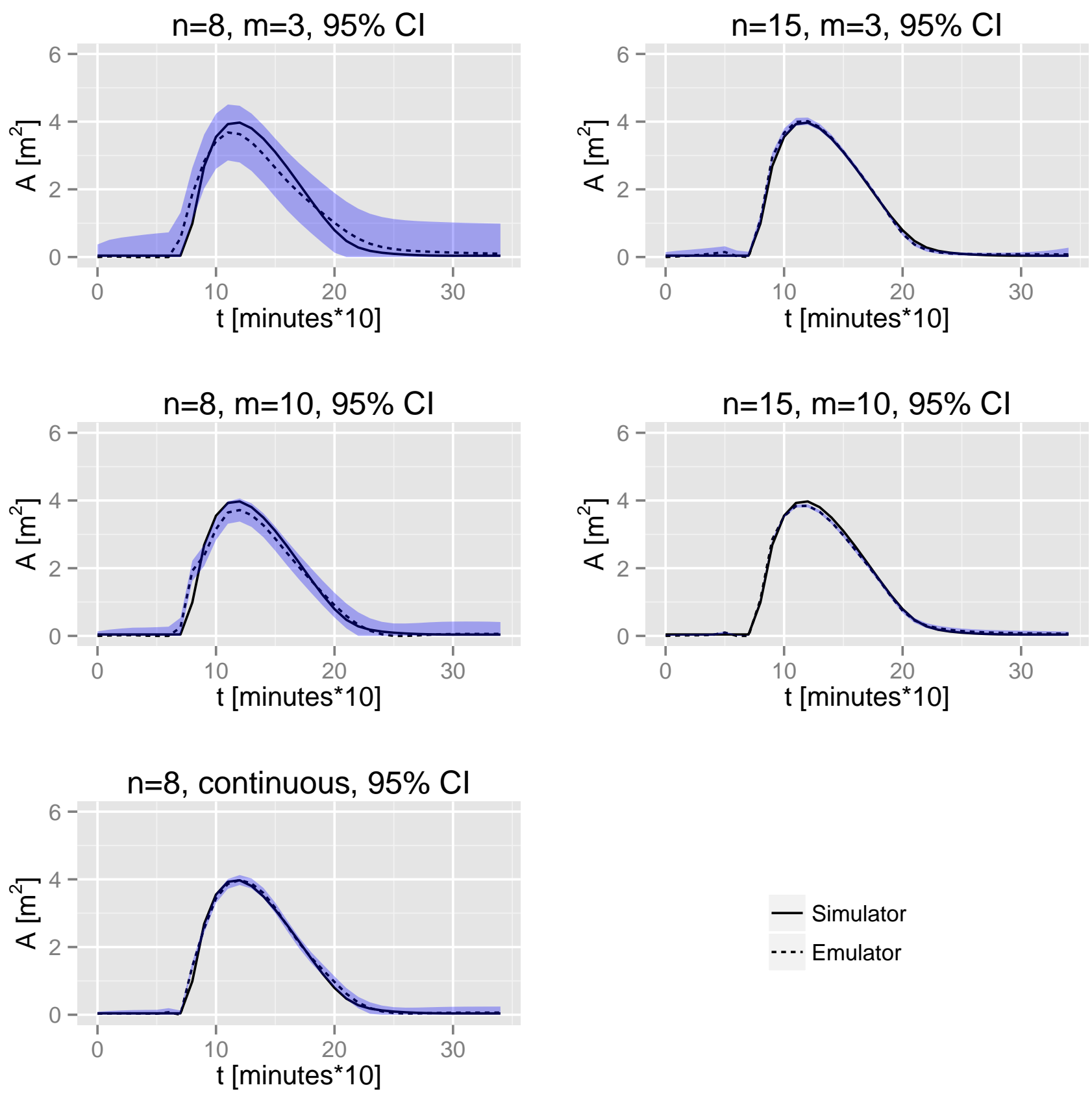

Figure 3: Comparison of the emulator uncertainty, with 95\% confidence intervals shown, for various amounts of (randomly selected) design data sets $n$ and of various model complexities $m$. The confidence intervals are not always reliable, but here they help us to see how the improved mechanisms reduce the uncertainty of the emulator (left column). For more design data sets (right column), this effect is not that prominent. These outputs were emulated for the following parameter values: $n_{M}=0.062 \mathrm{~s} / \mathrm{m}^{1 / 3}, w=0.82 \mathrm{~m}, L=695 \mathrm{~m}$ and $d=16.9 \mathrm{~m}$ with the resulting RMSE (column-wise, from the top left corner): $0.263,0.214,0.101,0.072,0.065$ (all in $\mathrm{m}^{2}$ ). 


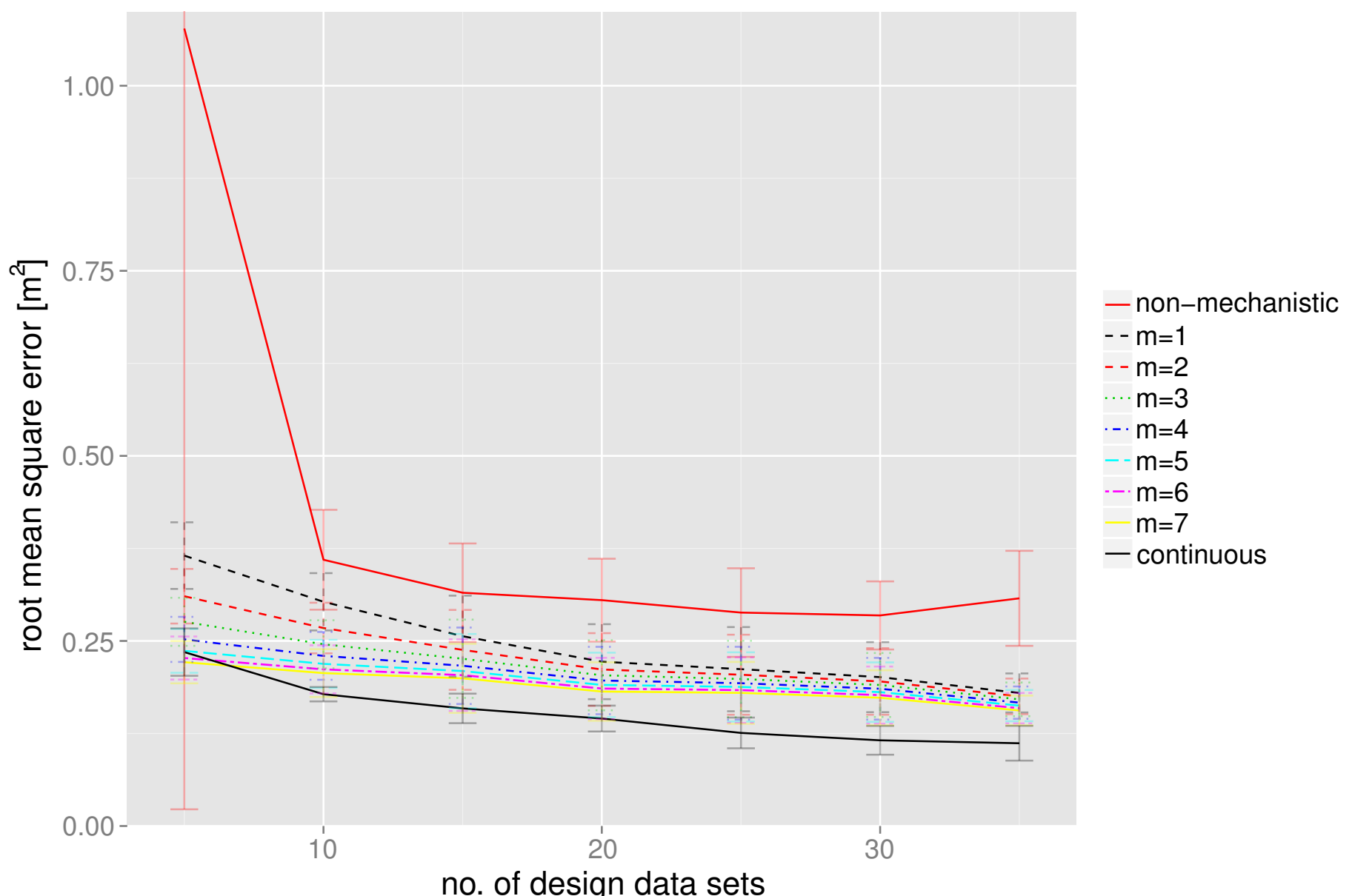

Figure 4: The dependency of the root mean squared error of the emulator on the number of design data sets and on the level of discretization. For each amount of randomly selected design data sets (selected out of 100 design data sets), the emulation was done for 20 test data sets (selected randomly out of other 100 data sets) and the root mean squared error of the emulated test data sets with respect to the simulated test data sets was calculated. This was done 10 times and averaged, with standard deviation of this averaging plotted as the error bars. The root mean squared error for the model complexity 0 corresponds to a non-mechanistic Gaussian emulator. Except for this case and the continuous case, the model complexity represents what is denoted by $m$ in the text. 
In Figure 3, emulator outputs with their confidence intervals are shown, for various model complexities and amounts $n$ of design data sets, with parameters $k_{0}, k_{1}$ and $\mu$ again estimated for each case. The confidence intervals provide an estimate of the uncertainty of the emulator, but they are not always reliable.

For the discrete case, we have in the emulation step (38) $\mathcal{O}\left(N_{t} n\right)$ (since $N_{x}=1$ in our case) multiplications of matrices of dimension $m$, where matrix multiplication is of complexity $\mathcal{O}\left(\mathrm{m}^{3}\right)^{3}$. The complexity of the emulation step therefore grows almost cubically with increasing $m$, linearly with the number of design data sets and linearly with the number of time-points.

\section{Summary and Conclusions}

We extended the mechanism-based emulation methodology of dynamic simulators from discrete time, discrete space [14] and continuous time, discrete space [15] approaches to a continuous time, continuous space approach. This leads to a more straightforward construction of mechanism-based emulators for simulators that implement partial differential equation models.

In a case study that uses a simulator which implements the diffusive approximation of the shallow water equations to describe discharge and water level in channels, we constructed a series of emulators that consider the mechanisms underlying the simulator to different degrees. These emulators range from a non mechanism-based emulator, over emulators based on spatial discretizations of the partial differential equation, to an emulator based on the new, continuous state space approach. This set of emulators allowed us to compare the gain in emulation accuracy when considering mechanisms to the gain in accuracy when increasing the design data set. In our case study, we could demonstrate that the emulation accuracy increased considerably already when considering the mechanisms to the lowest degree possible (by approximating the channel by a single linear reservoir). Further increases in complexity, by considering multiple reservoirs in sequence, led only to a minor gain in accuracy, but decreased the computational efficiency of the emulator. Constructing the emulator based on a simplified model with many reservoirs in series is thus not an efficient emulation strategy for our case study. To consider the mechanisms to a larger degree, it is more advisable to use the new, continuousspace approach for constructing the emulator. This emulator led again to a similar improvement of emulation accuracy compared to the emulator with a few reservoirs as was achieved with that emulator compared to the one that does not consider mechanisms. Furthermore, for this case study, this new emulator is faster than a multi-reservoir emulator if the number of reservoirs exceeds 5 . The decision whether to use the discrete-space approach with a single linear reservoir or the continuousspace approach is ultimately a trade-off between the desired accuracy of the emulator and the available computational resources.

The results of this paper indicate that for computationally very demanding simulators, for which accuracy gains through the enlargement of the design data set are difficult to realize, it may be useful to use a mechanism-based emulation strategy. The drawback of this strategy is that a specific emulator has to be constructed for each model which requires a thoughtful construction of a linear, approximating model. However, if the results of our case study extend to other cases, already a very crude consideration of mechanisms is sufficient to obtain a considerable gain in accuracy. This would limit the case-specific burden of this approach.

\section{Acknowledgements:}

This work is part of the project "Using Commercial Microwave Links and Computer Model Emulation to Reduce Uncertainties in Urban Drainage Simulations" (COMCORDE) led by Dr. Jörg

\footnotetext{
${ }^{3}$ Recent development by Williams [34] allows for matrix multiplication of complexity $\mathcal{O}\left(\mathrm{m}^{2.373}\right)$.
} 
Rieckermann and funded by the Swiss National Science Foundation, grants no. CR22I2 135551 and CR22I2 152824. The authors would like to thank him and other participants in the project, namely

Andreas Scheidegger and Dario Del Giudice, for stimulating discussions and inputs. This paper also profited from the stimulating comments by the reviewers.

\section{References}

[1] A. O'Hagan, Bayesian analysis of computer code outputs: A tutorial, Reliability Engineering and System Safety 91 (2006) 1290-1300.

[2] C. Currin, T. Mitchell, M. Morris, D. Ylvisaker, Bayesian prediction of deterministic functions, with applications to the design and analysis of computer experiments, Journal of the American Statistical Association 86 (416) (1991) 953-963.

[3] A. O'Hagan, Some bayesian numerical analysis, Bayesian statistics 4 (345-363) (1992) 4-2.

[4] T. J. Santner, B. J. Williams, W. I. Notz, The design and analysis of computer experiments, Springer Science \& Business Media, 2013.

[5] M. J. Bayarri, J. O. Berger, R. Paulo, J. Sacks, J. A. Cafeo, J. Cavendish, C.-H. Lin, J. Tu, A framework for validation of computer models, Technometrics 49 (2) (2007) 138-154. doi: 10.1198/004017007000000092.

[6] M. C. Kennedy, A. O'Hagan, Bayesian calibration of computer models, Journal of the Royal Statistical Society. Series B (Statistical Methodology) 63 (3) (2001) 425-464.

[7] S. Conti, A. O'Hagan, Bayesian emulation of complex multi-output and dynamic computer models, Journal of Statistical Planning and Inference 140 (2010) 640-651.

[8] A. Castelletti, S. Galelli, M. Ratto, R. Soncini-Sessa, P. Young, A general framework for dynamic emulation modelling in environmental problems, Environmental Modelling \& Software 34 (0) (2012) 5-18. doi:http://dx.doi.org/10.1016/j.envsoft.2012.01.002.

[9] D. Higdon, J. Gattiker, B. Williams, M. Rightley, Computer model calibration using highdimensional output, Journal of the American Statistical Association 103 (482).

[10] M. J. Bayarri, J. O. Berger, J. Cafeo, G. Garcia-Donato, F. Liu, J. Palomo, R. J. Parthasarathy, R. Paulo, J. Sacks, D. Walsh, Computer model validation with functional output, Annals of Statistics 35 (5) (2007) 1874-1906.

[11] S. Bhattacharya, A simulation approach to Bayesian emulation of complex dynamic computer models, Bayesian analysis 2 (4) (2007) 783-816.

[12] S. Conti, J. P. Gosling, J. E. Oakley, A. O'Hagan, Gaussian process emulation of dynamic computer codes, Biometrika 96 (3) (2009) 663-676. doi:10.1093/biomet/asp028.

[13] F. Liu, M. West, A dynamic modelling strategy for Bayesian computer model emulation, Bayesian analysis 4 (2009) 393-412.

[14] P. Reichert, G. White, M. J. Bayarri, E. B. Pitman, Mechanism-based emulation of dynamic simulation models: Concept and application in hydrology, Computational Statistics \& Data Analysis 55 (4) (2011) 1638-1655. doi:10.1016/j.csda.2010.10.011.

[15] C. Albert, A mechanistic dynamic emulator, Nonlinear Analysis: Real World Applications 13 (6) (2012) 2747 - 2754. doi:10.1016/j.nonrwa.2012.04.003. 
[16] EPA, Storm water management model, http://www.epa.gov/nrmrl/wswrd/wq/models/swmm/, [Online; accessed 15-May-2013] (2013).

[17] C. E. Rasmussen, C. K. I. Williams, Gaussian Processes for Machine Learning, The MIT Press, 2006, Ch. Covariance Functions, pp. 79-104.

[18] C. G. Kaufman, D. Bingham, S. Habib, K. Heitmann, J. A. Frieman, Efficient emulators of computer experiments using compactly supported correlation functions, with an application to cosmology, Ann. Appl. Stat. 5 (4) (2011) 2470-2492. doi:10.1214/11-A0AS489.

[19] H. Kleinert, Path Integrals in Quantum Mechanics, Statistics, Polymer Physics, and Financial Markets, EBL-Schweitzer, World Scientific Publishing Company, Incorporated, 2009. URL http://books.google.com/books?id=VJ1qNz5xYzkC

[20] W. L. Goffe, G. D. Ferrier, J. Rogers, Simann: Fortran module to perform global optimization of statistical functions with simulated annealing (1992).

[21] H. Ltaief, S. Tomov, R. Nath, P. Du, J. Dongarra, A scalable high performant Cholesky factorization for multicore with GPU accelerators, in: J. Palma, M. Dayd, O. Marques, J. Lopes (Eds.), High Performance Computing for Computational Science VECPAR 2010, Vol. 6449 of Lecture Notes in Computer Science, Springer Berlin Heidelberg, 2011, pp. 93-101. doi: 10.1007/978-3-642-19328-6_11.

[22] M. Anitescu, J. Chen, L. Wang, A matrix-free approach for solving the parametric Gaussian process maximum likelihood problem, SIAM J. Sci. Comput. 34 (1) (2012) 240-262. doi:10. $1137 / 110831143$.

[23] M. de St. Venant, Théorie du mouvement non permanant des eaux crues des rivières et à l'introduction des marées dans leur lit, Comptes Rendus 73 (1871) 147-154 \& 237-240.

[24] V. T. Chow, Open-channel hydraulics, McGraw-Hill, New York, 1959.

[25] F. M. Henderson, Open channel flow, Macmillan, New York, 1966.

[26] B. C. Yen, Open-channel flow equations revisited, Journal of the Mechanical Engineering Division, ASCE 99 (1973) 979-1009.

[27] B. C. Yen, Unsteady flow mathematical modeling techniques, in: H. Shen (Ed.), Modeling of Rivers, John Wiley, New York, 1979, pp. 13-1-13-33.

[28] R. H. French, Open-channel hydraulics, McGraw-Hill, Stuttgart, 1985, new York.

[29] R. Manning, On the flow of water in open channels and pipes, Transactions of the Institution of Civil Engineers of Ireland 20 (1891) 161-207.

[30] I. Wolfram Research, Mathematica, version 10.2 Edition, Wolfram Research, Inc., Champaign, Illinois, 2015.

[31] K. Watanabe, Greens functions for Laplace and wave equations, in: Integral Transform Techniques for Green's Function, Vol. 71 of Lecture Notes in Applied and Computational Mechanics, Springer International Publishing, 2014, pp. 11-42. doi:10.1007/978-3-319-00879-0_2.

[32] J. Oakley, A. O'Hagan, Bayesian inference for the uncertainty distribution of computer model outputs, Biometrika 89 (4) (2002) 769-784. 
[33] R. K. S. Hankin, Introducing BACCO, an R bundle for Bayesian analysis of computer code output, Journal of Statistical Software 14.

[34] V. V. Williams, Breaking the Coppersmith-Winograd barrier, Unpublished manuscript. URL http://www.cs.rit.edu/ rlc/Courses/Algorithms/Papers/matrixMult.pdf 